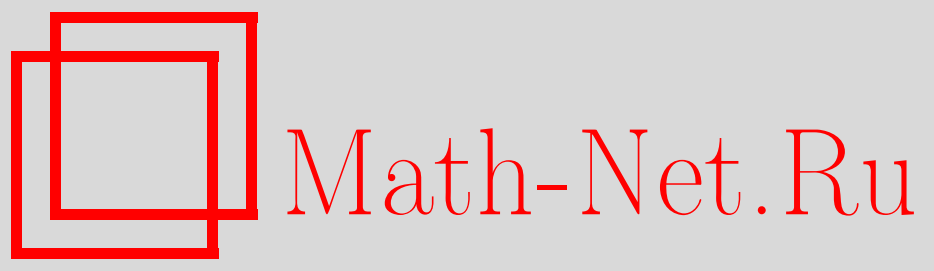

М. Бойти, Ф. Пемпинелли, А. К. Погребков, Свойства солитонных потенциалов оператора теплопроводности, TMФ, 2011, том 168, номер 1, 13-23

DOI: https://doi.org/10.4213/tmf6660

Использование Общероссийского математического портала Math-Net.Ru подразумевает, что вы прочитали и согласны с пользовательским соглашением http://www . mathnet.ru/rus/agreement

Параметры загрузки:

IP : 54.196 .121 .252

26 апреля 2023 г., $17: 29: 56$

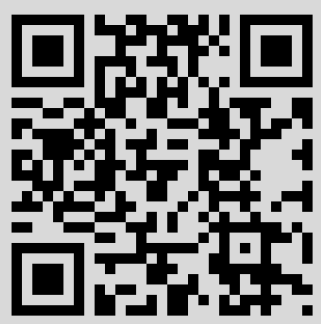




\title{
СВОЙСТВА СОЛИТОННЫХ ПОТЕНЦИАЛОВ ОПЕРАТОРА ТЕПЛОПРОВОДНОСТИ
}

\begin{abstract}
Подробно рассмотрены свойства чисто солитонных тау-функций и потенциала уравнения теплопроводности. Описано асимптотическое поведение потенциала и установлена зависимость лучевой структуры этого асимптотического поведения на плоскости $\left(x_{1}, x_{2}\right)$ от параметров потенциала.
\end{abstract}

Ключевые слова: уравнение Кадомцева-Петвиашвили, солитоны, асимптотика потенциала.

\section{1. ВВЕДЕНИЕ}

Уравнение Кадомцева-Петвиашвили (КП) было выведено как модель слабо двумерных волн с малой амплитудой и большой длиной волны в слабо диспергирующей среде [1]. Это уравнение есть $(2+1)$-мерное обобщение знаменитого уравнения Кортевега-де Фриза (КдФ), и с начала 70-х годов известно [2], [3], что оно интегрируемо. Существуют две неэквивалентные версии уравнения КП: уравнения КП-I и KП-II. В настоящей работе мы рассматриваем уравнение КП-II

$$
\left(u_{t}-6 u u_{x_{1}}+u_{x_{1} x_{1} x_{1}}\right)_{x_{1}}=-3 u_{x_{2} x_{2}}
$$

(уравнение КП-І имеет знак плюс в правой части), где $u=u(x, t), x=\left(x_{1}, x_{2}\right)$, индексы $x_{1}, x_{2}$ и $t$ означают частные производные. Уравнение КП-ІІ интегрируемо, поскольку может быть представлено как условие совместности $[\mathcal{L}, \mathcal{T}]=0$ для пары Лакса $\mathcal{L}$ и $\mathcal{T}$, где оператор

$$
\mathcal{L}\left(x, \partial_{x}\right)=-\partial_{x_{2}}+\partial_{x_{1}}^{2}-u(x)
$$

задает хорошо известное уравнение теплопроводности, а оператор $\mathcal{T}$ имеет вид

$$
\mathcal{T}\left(x, \partial_{x}, \partial_{t}\right)=\partial_{t}+4 \partial_{x_{1}}^{3}-6 u \partial_{x_{1}}-3 u_{x_{1}}-3 \partial_{x_{1}}^{-1} u_{x_{2}}
$$

Спектральная теория оператора (1.2) в случае вещественного потенциала $u(x)$, быстро убывающего на пространственной бесконечности, была развита в рабо-

*Dipartimento di Fisica, Università del Salento and Sezione INFN, Lecce, Italy

${ }^{\dagger}$ Математический институт им. В. А. Стеклова РАН, Москва, Россия. E-mail: pogreb@mi.ras.ru 
тах [4]-[7]. Однако этот случай не является наиболее интересным, поскольку уравнение КП-ІІ было предложено в работе [1] именно для рассмотрения слабого двумерного поперечного возмущения односолитонного решения уравнения КдФ. Действительно, если функция $u_{1}\left(t, x_{1}\right)$ удовлетворяет уравнению КдФ, то функция $u\left(t, x_{1}, x_{2}\right)=u_{1}\left(t, x_{1}+\mu x_{2}-3 \mu^{2} t\right)$ является решением уравнения KП-II для произвольной константы $\mu \in \mathbb{R}$. В частности, уравнение KП-II допускает односолитонное решение вида

$$
u(x, t)=-\frac{\left(\kappa_{1}-\kappa_{2}\right)^{2}}{2} \operatorname{sech}^{2}\left[\frac{\kappa_{1}-\kappa_{2}}{2} x_{1}+\frac{\kappa_{1}^{2}-\kappa_{2}^{2}}{2} x_{2}-2\left(\kappa_{1}^{3}-\kappa_{2}^{3}\right) t\right],
$$

где $\kappa_{1}$ и $\kappa_{2}$ суть вещественные произвольные константы.

Спектральная теория оператора теплопроводности (1.2), включающая также и солитоны, еще ждет своего построения. В случае потенциала $u(x)$, быстро спадающего на пространственной бесконечности, согласно работам [4]-[7] основными инструментами построения спектральной теории оператора (1.2), как и в одномерном случае, являются интегральные уравнения, задающие решения Йоста. Однако, если потенциал $u(x)$ не убывает на пространственной бесконечности, как в случае солитонных потенциалов (см., например, решение (1.4)), эти интегральные уравнения плохо определены, поэтому необходим более общий подход. В ходе решения аналогичной проблемы для нестационарного оператора Шредингера, ассоциированного с уравнением КП-I, был введен метод расширенной резольвенты [8]. Соответственно, спектральную теорию уравнения КП-II, также включающую солитоны, следует изучать с помощью резольвентного подхода. На этой основе оказалось возможным развить метод обратной задачи для решения, описывающего один солитон на произвольном быстроубывающем фоне [9], и исследовать существование (расширенной) резольвенты для (некоторых) многосолитонных решений [10].

Однако общий случай $N$ солитонов остается открытым. Это обусловлено, в частности, сложным асимптотическим поведением чисто солитонных потенциалов на плоскости $\left(x_{1}, x_{2}\right)$. В настоящей работе мы даем детальное описание этого поведения в терминах солитонных параметров. Общая форма многосолитонного потенциала была выведена посредством рациональных преобразований данных рассеяния в работе [11] и посредством сплетающего преобразования в работе [10]. В работе [12] мы доказали, что результаты обеих этих конструкций совпадают друг с другом, а также с формой многосолитонных потенциалов, полученной в терминах тау-функции (см. обзорную статью [13] и приведенные в ней ссылки).

Статья организована следующим образом. В разделе 2 мы приводим многосолитонные потенциалы. Они характеризуются двумя целыми числами (топологическими зарядами) $N_{a}$ и $N_{b}$, удовлетворяющими условию

$$
N_{a}, N_{b} \geqslant 1
$$

и имеют на больших расстояниях $N_{b}$ "входящих" лучей и $N_{a}$ "выходящих" лучей. Используя обозначения работы [12], мы даем для этих потенциалов два дуальных представления через тау-функции, зависящие от $\mathcal{N}$ вещественных параметров $\kappa_{n}$, где

$$
\mathcal{N}=N_{a}+N_{b}
$$


(так что $\mathcal{N} \geqslant 2)$, и от постоянной $\left(N_{a} \times N_{b}\right)$-матрицы $d$. В разделе 3 мы подробно исследуем асимптотическое поведение многосолитонных потенциалов при больших $x$ и показываем, что круговой угол в начале координат может быть разделен на $\mathcal{N}$ угловых секторов таких, что в направлениях ограничивающих их лучей потенциал имеет постоянное солитоноподобное поведение, в то время как по направлениям внутри секторов потенциал имеет экспоненциально убывающее поведение, которое мы приводим в явном виде. В частности, для специального подкласса солитонных потенциалов мы описываем структуру лучей явно в терминах параметров $\kappa_{n}$.

\section{2. МНОГОСОЛИТОННЫЕ ПОТЕНЦИАЛЫ}

Пусть заданы $\mathcal{N}$ вещественных параметров

$$
\kappa_{1}<\kappa_{2}<\cdots<\kappa_{\mathcal{N}}
$$

Введем функции

$$
K_{n}(x)=\kappa_{n} x_{1}+\kappa_{n}^{2} x_{2}, \quad n=1, \ldots, \mathcal{N} .
$$

Пусть $e^{K(x)}=\operatorname{diag}\left\{e^{K_{n}(x)}\right\}_{n=1}^{\mathcal{N}}-$ диагональная $(\mathcal{N} \times \mathcal{N})$-матрица, $\mathcal{D}$ - постоянная вещественная $\left(\mathcal{N} \times N_{b}\right)$-матрица с по крайней мере двумя ненулевыми максимальными минорами, а $\mathcal{V}$ - "неполная матрица Вандермонда", т. е. $\left(N_{b} \times \mathcal{N}\right)$-матрица вида

$$
\mathcal{V}=\left(\begin{array}{ccc}
1 & \ldots & 1 \\
\vdots & \ddots & \vdots \\
\kappa_{1}^{N_{b}-1} & \ldots & \kappa_{\mathcal{N}}^{N_{b}-1}
\end{array}\right)
$$

Тогда солитонный потенциал записывается как

$$
u(x)=-2 \partial_{x_{1}}^{2} \ln \tau(x),
$$

где тау-функция равна

$$
\tau(x)=\operatorname{det}\left(\mathcal{V} e^{K(x)} \mathcal{D}\right)
$$

(см. обзорную статью [13], ссылки в ней, а также работу [12], где использованы те же обозначения).

Существует (см. статьи [10], [12], [14]) дуальное представление для потенциала в терминах тау-функции:

$$
\tau^{\prime}(x)=\operatorname{det}\left(\mathcal{D}^{\prime} e^{-K(x)} \gamma \mathcal{V}^{\prime}\right)
$$

Здесь $\mathcal{D}^{\prime}$ - постоянная вещественная $\left(N_{a} \times \mathcal{N}\right)$-матрица, которая подобно матрице $\mathcal{D}$ имеет по крайней мере два ненулевых максимальных минора и ортогональна матрице $\mathcal{D}$ в том смысле, что

$$
\mathcal{D}^{\prime} \mathcal{D}=0
$$

причем ноль в правой части - это $\left(N_{a} \times N_{b}\right)$-матрица. В формуле $(2.6)$ матрица $\mathcal{V}^{\prime}$ - это $\left(\mathcal{N} \times N_{b}\right)$-матрица вида

$$
\mathcal{V}^{\prime}=\left(\begin{array}{ccc}
1 & \ldots & \kappa_{1}^{N_{a}-1} \\
\vdots & \ddots & \vdots \\
1 & \ldots & \kappa_{\mathcal{N}}^{N_{a}-1}
\end{array}\right)
$$


а $\gamma$ - постоянная диагональная вещественная $(\mathcal{N} \times \mathcal{N})$-матрица:

$$
\gamma=\operatorname{diag}\left\{\gamma_{n}\right\}_{n=1}^{\mathcal{N}}, \quad \gamma_{n}=\prod_{\substack{n^{\prime}=1, n^{\prime} \neq n}}^{\mathcal{N}}\left(\kappa_{n}-\kappa_{n^{\prime}}\right)^{-1}
$$

Дуальное представление получается, если заметить, что

$$
\tau(x)=(-1)^{N_{a} N_{b}+N_{a}\left(N_{a}-1\right) / 2}\left(\prod_{n=1}^{\mathcal{N}} e^{K_{n}(x)}\right) V\left(\kappa_{1}, \ldots, \kappa_{N_{a}+N_{b}}\right) \tau^{\prime}(x),
$$

где $V$ означает детерминант Вандермонда,

$$
V\left(\kappa_{1}, \ldots, \kappa_{\mathcal{N}}\right)=\prod_{1 \leqslant m<n \leqslant \mathcal{N}}\left(\kappa_{n}-\kappa_{m}\right) .
$$

Матрицы $\mathcal{D}$ и $\mathcal{D}^{\prime}$ обладают довольно интересными свойствами [12]. Принимая во внимание, что матрица $\mathcal{D}$ имеет ненулевые максимальные миноры, мы всегда можем записать ее в виде $\mathcal{D}=\pi\left(\begin{array}{l}d_{1} \\ d_{2}\end{array}\right)$, где $\pi-(\mathcal{N} \times \mathcal{N})$-матрица перестановки такая, что $\left(N_{b} \times N_{b}\right)$-матрица $d_{2}$ несингулярна. Тогда мы можем переписать последнее равенство в виде

$$
\mathcal{D}=\pi\left(\begin{array}{c}
d \\
E_{N_{b}}
\end{array}\right) d_{2}
$$

где $d=d_{1} d_{2}^{-1}-$ постоянная вещественная $\left(N_{a} \times N_{b}\right)$-матрица, а $E_{N_{b}}-$ единичная $\left(N_{b} \times N_{b}\right)$-матрица.

Заметим (см. [13]), что выражение для потенциала в (2.4), равно как и условие (2.7), остаются неизменными, если соответственно в (2.5) и (2.6) мы умножим $\left(\mathcal{N} \times N_{b}\right)$-матрицу $\mathcal{D}$ справа на несингулярную постоянную $\left(N_{b} \times N_{b}\right)$-матрицу, а $\left(N_{a} \times \mathcal{N}\right)$-матрицу $\mathcal{D}^{\prime}$ - слева на несингулярную постоянную $\left(N_{a} \times N_{a}\right)$-матрицу. Мы заключаем, что матрица $d_{2}$ несущественна, и матрица $\mathcal{D}$ может быть выбрана имеющей простую блочную структуру. Понятно, однако, что выбор матриц $\pi$ и $d$ не единствен, поскольку матрица $\mathcal{D}$ может иметь различные ненулевые максимальные миноры.

Запишем теперь $\mathcal{D}^{\prime} \pi=\left(d_{1}^{\prime} d_{2}^{\prime}\right)$, где $d_{1}^{\prime}-\left(N_{a} \times N_{a}\right)$-матрица и $d_{2}^{\prime}-\left(N_{a} \times N_{b}\right)$-матрица. Тогда в силу (2.7) мы имеем $d_{2}^{\prime}=-d_{1}^{\prime} d$, где $d$ - та же $\left(N_{a} \times N_{b}\right)$-матрица, что и в (2.12). Итак, для матрицы $\mathcal{D}^{\prime}$ мы получаем блочную структуру,

$$
\mathcal{D}^{\prime}=d_{1}^{\prime}\left(E_{N_{a}}-d\right) \pi^{\dagger},
$$

и благодаря эквивалентности $\tau$ и $\tau^{\prime}$ имеем, что $\operatorname{det} d_{1}^{\prime} \neq 0$, а тогда и $\mathcal{D}^{\prime}$ может быть выбрана имеющей простую блочную структуру.

Более того, вследствие блочной структуры матриц $\mathcal{D}$ и $\mathcal{D}^{\prime}$, данной в $(2.12)$ и $(2.13)$, мы получаем, что

$$
\mathcal{D}^{\dagger} \mathcal{D}=d_{2}^{\dagger}\left(E_{N_{b}}+d^{\dagger} d\right) d_{2}, \quad \mathcal{D}^{\prime} \mathcal{D}^{\prime \dagger}=d_{1}^{\prime}\left(E_{N_{a}}+d d^{\dagger}\right) d_{1}^{\prime \dagger},
$$

где $\dagger$ означает эрмитово сопряжение матрицы (на самом деле, здесь транспонирование). Итак, обе матрицы $\mathcal{D}^{\dagger} \mathcal{D}$ и $\mathcal{D}^{\prime} \mathcal{D}^{\prime \dagger}$ положительны, а потому $\mathcal{D}$ и $\mathcal{D}^{\prime}$ имеют соответственно левые и правые обратные [15]. А именно,

$$
(\mathcal{D})^{(-1)} \mathcal{D}=E_{N_{b}}, \quad \mathcal{D}^{\prime}\left(\mathcal{D}^{\prime}\right)^{(-1)}=E_{N_{a}}
$$


причем

$$
(\mathcal{D})^{(-1)}=\left(\mathcal{D}^{\dagger} \mathcal{D}\right)^{-1} \mathcal{D}^{\dagger}, \quad\left(\mathcal{D}^{\prime}\right)^{(-1)}=\mathcal{D}^{\prime \dagger}\left(\mathcal{D}^{\prime} \mathcal{D}^{\prime \dagger}\right)^{-1} .
$$

Произведения матриц в обратном порядке дают вещественные эрмитовы $(\mathcal{N} \times \mathcal{N})$ матрицы

$$
\begin{aligned}
P & =\mathcal{D}(\mathcal{D})^{(-1)}=\mathcal{D}\left(\mathcal{D}^{\dagger} \mathcal{D}\right)^{-1}(\mathcal{D})^{\dagger} \\
P^{\prime} & =\left(\mathcal{D}^{\prime}\right)^{(-1)} \mathcal{D}^{\prime}=\left(\mathcal{D}^{\prime}\right)^{\dagger}\left(\mathcal{D}^{\prime} \mathcal{D}^{\prime \dagger}\right)^{-1} \mathcal{D}^{\prime}
\end{aligned}
$$

которые являются ортогональными проекторами, т. е. $P^{2}=P,\left(P^{\prime}\right)^{2}=P^{\prime}$ и $P P^{\prime}=$ $0=P^{\prime} P$, дополнительными в том смысле, что $P+P^{\prime}=E_{\mathcal{N}}$. Взаимная ортогональность проекторов следует из (2.7), а последнее равенство вытекает из очевидных соотношений типа $\left(E_{N_{b}}+d^{\dagger} d\right)^{-1} d^{\dagger}=d^{\dagger}\left(E_{N_{a}}+d d^{\dagger}\right)^{-1}$. Заметим, наконец, что если все максимальные миноры матрицы $\mathcal{D}$ отличны от нуля, то перестановка $\pi$ в $(2.12)$ и (2.13) может быть выбрана произвольной, например ее можно взять тождественной, что дает упрощенные представления

$$
\mathcal{D}=\left(\begin{array}{c}
d \\
E_{N_{b}}
\end{array}\right) d_{2}, \quad \mathcal{D}^{\prime}=d_{1}^{\prime}\left(\begin{array}{cc}
E_{N_{a}} & -d
\end{array}\right),
$$

так что в данном частном случае без потери общности можно выбрать

$$
\mathcal{D}=\left(\begin{array}{c}
d \\
E_{N_{b}}
\end{array}\right), \quad \mathcal{D}^{\prime}=\left(\begin{array}{ll}
E_{N_{a}} & -d
\end{array}\right) .
$$

Для изучения свойств потенциала удобно использовать явное представление определителя (2.5). Используя формулу Бине-Коши для определителя произведения матриц, мы получаем

$$
\tau(x)=\sum_{1 \leqslant n_{1}<\cdots<n_{N_{b}} \leqslant \mathcal{N}} f_{n_{1}, \ldots, n_{N_{b}}} \prod_{l=1}^{N_{b}} e^{K_{n_{l}}(x)},
$$

где коэффициенты $f_{n_{1}, \ldots, n_{N_{b}}}$ записываются как

$$
f_{n_{1}, \ldots, n_{N_{b}}}=V\left(\kappa_{n_{1}}, \ldots, \kappa_{n_{N_{b}}}\right) \mathcal{D}\left(n_{1}, \ldots, n_{N_{b}}\right) .
$$

Здесь мы использовали определитель Вандермонда (2.11) и обозначение

$$
\mathcal{D}\left(n_{1}, \ldots, n_{N_{b}}\right)=\operatorname{det}\left(\begin{array}{ccc}
\mathcal{D}_{n_{1}, 1} & \ldots & \mathcal{D}_{n_{1}, N_{b}} \\
\vdots & \ddots & \vdots \\
\mathcal{D}_{n_{N_{b}}, 1} & \ldots & \mathcal{D}_{n_{N_{b}}, N_{b}}
\end{array}\right)
$$

для максимальных миноров матрицы $\mathcal{D}$. Отметим, что эти коэффициенты инвариантны относительно перестановки индексов.

Из (2.17) непосредственно следует, что условие

$$
f_{n_{1}, \ldots, n_{N_{b}}} \geqslant 0, \quad 1 \leqslant n_{1}<\cdots<n_{N_{b}} \leqslant \mathcal{N}
$$

достаточно [13] для регулярности потенциала $u(x)$, т. е. для отсутствия нулей функции $\tau(x)$ на плоскости $\left(x_{1}, x_{2}\right)$. В силу $(2.1),(2.11)$ и (2.18) это условие эквивалентно 
тому, что все максимальные миноры матрицы $\mathcal{D}$ неотрицательны. В работе [12] отмечалось, что условие (2.20) также необходимо для регулярности потенциала при эволюции по произвольному числу высших времен иерархии КП. В работе [13] было предложено разбить солитонные решения уравнения КП-II на подклассы, ассоциированные с ячейками Шуберта на грассманиане, и доказано, что условие (2.20) необходимо для регулярности всех решений, ассоциированных с одной ячейкой. Однако проблема нахождения условий, необходимых для регулярности многосолитонного решения при эволюции только по одному времени, отвечающему уравнению КП-II, все еще открыта.

Если матрица $\mathcal{D}$ может быть выбрана, как в $(2.16)$, то, вводя новую $\left(N_{a} \times N_{b}\right)$-матрицу $\widetilde{d}$ с элементами

$$
\widetilde{d}_{n, l}=d_{N_{a}+1-n, l}(-1)^{l+1}, \quad n=1, \ldots, N_{a}, \quad l=1, \ldots, N_{b},
$$

мы легко получаем с учетом (2.19), что

$$
\begin{gathered}
\mathcal{D}\left(n_{1}, \ldots, n_{N_{b}}\right)=\operatorname{det}\left\|\widetilde{d}_{n_{i}, l}\right\|, \\
i=1, \ldots, k, \quad l=1, \ldots, N_{b}, \quad l \neq n_{j}-N_{a}, \quad j=k+1, \ldots, N_{b},
\end{gathered}
$$

где номер $k, 1 \leqslant k \leqslant N_{b}$, определяется условием $n_{k} \leqslant N_{a}<n_{k+1}$. Это доказывает, что если мы налагаем условие $(2.20)$ на коэффициенты $f_{n_{1}, \ldots, n_{N_{b}}}$, то $\widetilde{d}$ - совершенно неотрицательная (положительная, если все неравенства в (2.20) строгие) матрица [16], [17].

Для дальнейших рассуждений удобно продолжить индексы величин $\kappa_{n}, f_{n_{1}, \ldots, n_{N_{b}}}$ и пр. периодически по $\mathcal{N}$ на всё $\mathbb{Z}$ условием

$$
n \longrightarrow n(\bmod \mathcal{N})
$$

Тогда равенство (2.17) можно записать в виде

$$
\tau(x)=\sum_{n \leqslant n_{1}<\cdots<n_{N_{b}} \leqslant \mathcal{N}+n-1} f_{n_{1}, \ldots, n_{N_{b}}} \prod_{l=1}^{N_{b}} e^{K_{n_{l}}(x)},
$$

где правая часть не зависит от $n$, а коэффициенты $f_{n_{1}, \ldots, n_{N_{b}}}$ определены по формуле (2.18) с учетом (2.21). Благодаря (2.1) и (2.21) мы можем доказать следующую лемму.

ЛЕмма 1. Пусть

$$
g_{l, m, n}=\left(\kappa_{l}-\kappa_{m}\right)\left(\kappa_{n}-\kappa_{n+N_{b}}\right)\left(\kappa_{l}+\kappa_{m}-\kappa_{n}-\kappa_{n+N_{b}}\right) .
$$

Тогда

$$
g_{l, m, n} \geqslant 0, \quad n \in \mathbb{Z}, \quad l=n, \ldots, n+N_{b}, \quad m=n+N_{b}, \ldots, \mathcal{N}+n,
$$

причем равенство имеет место только тогда, когда l и т независимо принимают значения $n$ или $n+N_{b}$ по модулю $\mathcal{N}$. 
ДоКАЗАТЕЛьство. В силу (2.21) достаточно доказать лемму для $n=1, \ldots, \mathcal{N}$. Для этих значений $n$ параметры $\kappa_{n}$ упорядочены, как в (2.1). Для других значений упорядочение получается за счет перехода по формуле (2.21) к значениям, принадлежащим интервалу $1 \leqslant n \leqslant \mathcal{N}$. Итак, если $n=1, \ldots, N_{a}$, то $n, n+N_{b}$ и $l$ не превосходят $\mathcal{N}$ (см. (1.6)), но вместо интервала $n+N_{b} \leqslant m \leqslant \mathcal{N}+n$, данного в (2.24), мы имеем два интервала: $n+N_{b} \leqslant m \leqslant \mathcal{N}$ и $1 \leqslant m \leqslant n$. Тогда для данных значений $n, l$ при значениях $m$, лежащих в первом интервале, утверждение леммы выполнено, поскольку первый множитель в (2.23) неположителен, второй отрицателен, а третий неотрицателен. Первый множитель может быть равен нулю только при $l=m$, и тогда $l=m=n+N_{b}$, а третий множитель равен нулю только при $l=n$ и $m=n+N_{b}$ (все равенства по модулю $\left.\mathcal{N}\right)$. Для $m$, лежащих во втором интервале, первый множитель неотрицателен (равен нулю только при $l=m=n(\bmod \mathcal{N}))$, второй множитель отрицателен, а третий множитель неположителен (равен нулю только при $l=n+N_{b}$ и $\left.m=n\right)$. В интервале значений $N_{a}+1 \leqslant n \leqslant \mathcal{N}$ мы имеем с учетом (2.21) $\kappa_{n+N_{b}}=\kappa_{n-N_{a}}$, так что снова $n$ и $n-N_{a}$ принадлежат интервалу $1, \ldots, \mathcal{N}$. Теперь интервал значений $n \leqslant l \leqslant n+N_{b}$, данный в $(2.24)$, распадается на два интервала $n \leqslant l \leqslant \mathcal{N}$ и $1 \leqslant l \leqslant n-N_{a}$, в то время как $m=n-N_{a}, \ldots, n$, так что $m$ положительно и меньше $\mathcal{N}$. Тогда для этих значений $n, m$ при значениях $l$, лежащих в первом интервале, благодаря (2.1) первый множитель в (2.23) неотрицателен (равен нулю только при $l=m=n(\bmod \mathcal{N}))$, второй множитель положителен, а третий неотрицателен (равен нулю только при $l=n$ и $m=n+N_{b}$ ). В случае второго интервала значений $l$ первый множитель неположителен (равен нулю только при $\left.l=m=n-N_{a}=n+N_{b}(\bmod \mathcal{N})\right)$, второй положителен, а третий неположителен (равен нулю только при $l=n-N_{a}=n+N_{b}(\bmod \mathcal{N})$ и $\left.m=n\right)$. Лемма доказана.

\section{3. АСИМПТОТИЧЕСКОЕ ПОВЕДЕНИЕ ТАУ-ФУНКЦИИ И ПОТЕНЦИАЛА $u(x)$}

Здесь мы рассмотрим асимптотическое поведение функции $\tau(x)$, которое в силу (2.17) определено соотношениями между функциями $K_{n}(x)$ при различных $n$. Вследствие (2.2) мы имеем

$$
K_{l}(x)-K_{m}(x)=\left(\kappa_{l}-\kappa_{m}\right)\left(x_{1}+\left(\kappa_{l}+\kappa_{m}\right) x_{2}\right)
$$

для любых $l, m \in \mathbb{Z}$, т. е. эти разности линейны по пространственным переменным, поэтому асимптотическое поведение должно иметь секторную структуру на плоскости $\left(x_{1}, x_{2}\right)$. Для описания этой структуры введем лучи

$$
r_{n}=\left\{x: x_{1}+\left(\kappa_{n+N_{b}}+\kappa_{n}\right) x_{2}=0,\left(\kappa_{n+N_{b}}-\kappa_{n}\right) x_{2}<0\right\}, \quad n=1, \ldots, \mathcal{N},
$$

которые пересекают линии $x_{2}=1$ и $x_{2}=-1$ в точках $\left(\kappa_{n+N_{b}}+\kappa_{n},-1\right), n=1, \ldots, N_{a}$, и в точках $\left(-\kappa_{n+N_{b}}-\kappa_{n}, 1\right), n=N_{a}+1, \ldots, \mathcal{N}$, соответственно. В силу (2.1) и (2.21) мы имеем $\kappa_{n+N_{b}}-\kappa_{n}>0$ при $n=1, \ldots, N_{a}$ и $\kappa_{n+N_{b}}-\kappa_{n}<0$ при $n=N_{a}+1, \ldots, \mathcal{N}$, а потому при возрастании $n$ от 1 до $N_{a}$ луч вращается против часовой стрелки в нижней полуплоскости плоскости $\left(x_{1}, x_{2}\right)$, пересекает положительную часть оси $x_{1}$ при подходе к $n=N_{a}+1$, а затем вращается против часовой стрелки в верхней полуплоскости плоскости $\left(x_{1}, x_{2}\right)$ до $n=\mathcal{N}$ и, наконец, пересекая отрицательную часть оси $x_{1}$, при $n=\mathcal{N}+1$ опять возвращается в луч $r_{1}$ в силу $(2.21)$. 


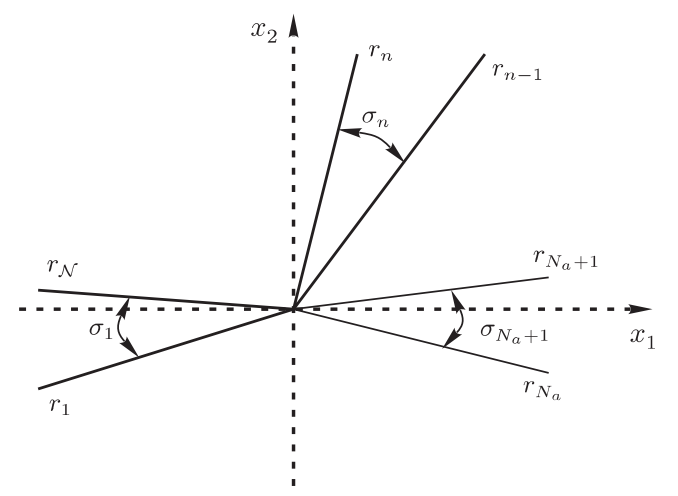

Секторы $\sigma_{n}$, соответствующие $N_{a}+2 \leqslant n \leqslant \lambda$.

Допустим, что некоторые лучи, скажем $r_{m}$ и $r_{n}$, где для определенности $m<n$, параллельны. По определению (3.2) это означает, что $\kappa_{m+N_{b}}+\kappa_{m}=\kappa_{n+N_{b}}+\kappa_{n}$, т. е. $\kappa_{n}-\kappa_{m}=\kappa_{m+N_{b}}-\kappa_{n+N_{b}}$, где левая часть равенства положительна по формуле (2.1). Тогда в силу (2.21) легко видеть, что правая часть может быть положительна, только если $1 \leqslant m \leqslant N_{a}<n \leqslant \mathcal{N}$, так что вследствие определения (3.2) луч $r_{m}$ лежит в нижней полуплоскости, а луч $r_{n}$ - в верхней. Итак, лучи могут быть параллельны, только если они принадлежат различным полуплоскостям, но в случае $N_{a} \neq N_{b}$ это возможно только при специальном выборе параметров $\kappa_{n}$. Напротив, в случае $N_{a}=N_{b}$ все пары $r_{n}$ и $r_{n+N_{a}}, n=1, \ldots, N_{a}$, и только эти пары дают параллельные лучи. В частном случае $N_{a}=N_{b}=1$ мы имеет два луча, дающих прямую линию $x_{1}+\left(\kappa_{1}+\kappa_{2}\right) x_{2}=0$, делящую плоскость $\left(x_{1}, x_{2}\right)$ на две полуплоскости.

Теперь мы вводим секторы $\sigma_{n}$ на плоскости $\left(x_{1}, x_{2}\right)$ :

$$
\sigma_{n}=\left\{x: K_{n-1}(x)<K_{n+N_{b}-1}(x), K_{n}(x)>K_{n+N_{b}}(x)\right\}, \quad n=1, \ldots, \mathcal{N} .
$$

В силу (3.1) и предыдущего обсуждения $\sigma_{n}$ являются секторами с острыми (при $\mathcal{N}>2$ ) углами и вершинами в начале координат; они ограничены справа (если смотреть из начала координат) лучом $r_{n-1}$ и слева лучом $r_{n}$. С ростом $n$ секторы $\sigma_{n}$ упорядочены против часовой стрелки, начиная “слева" с сектора $\sigma_{1}$, включающего отрицательную часть оси $x_{1}$, затем следуют секторы $\sigma_{n}, n=2, \ldots, N_{a}$, в нижней полуплоскости, сектор $\sigma_{N_{a}+1}$ "справа", включающий положительную часть оси $x_{1}$, и секторы $\sigma_{n}, n=N_{a}+2, \ldots, \mathcal{N}$, в верхней полуплоскости, заканчивающиеся сектором $\sigma_{\mathcal{N}}$, касательным сектору $\sigma_{1}$ (см. рисунок). Таким образом, указанные секторы покрывают образом всю плоскость $\left(x_{1}, x_{2}\right)$ за исключением ограничивающих лучей $r_{n}$. Поэтому секторы $\sigma_{n}$ определяют разбиение кругового угла в начале координат на $\mathcal{N}$ частей, причем $n$ играет роль дискретной угловой переменной. Очевидно, что при анализе асимптотического поведения тау-функции мы рассматриваем плоскость $\left(x_{1}, x_{2}\right)$ как векторное пространство, поскольку при $x \rightarrow \infty$ существенны только направления вектора $x=\left(x_{1}, x_{2}\right)$. Для фиксации направлений лучей $r_{n}$ и секторов $\sigma_{n}$ мы вводим векторы

$$
y_{n}=\left(\kappa_{n+N_{b}}^{2}-\kappa_{n}^{2}, \kappa_{n}-\kappa_{n+N_{b}}\right), \quad n=1, \ldots, \mathcal{N},
$$

позволяющие дать следующие определения. 
ОПРЕДЕЛЕНИЕ. Будем говорить, что $x \rightarrow \infty$ вдоль луча $r_{n}$, если $x \rightarrow \infty$ и существует такая функция $\alpha(x) \rightarrow+\infty$, что вектор $x-\alpha(x) y_{n}$ ограничен. Это будет обозначаться как $x \stackrel{r_{n}}{\longrightarrow} \infty$.

Будем говорить, что $x \rightarrow \infty$ в секторе $\sigma_{n}$, если $x \rightarrow \infty$ и существуют такие функции $\alpha(x) \rightarrow+\infty$ и $\beta(x) \rightarrow+\infty$, что вектор $x-\alpha(x) y_{n-1}-\beta(x) y_{n}$ ограничен. Это будет обозначаться как $x \stackrel{\sigma_{n}}{\longrightarrow} \infty$.

Отметим, что при $x \stackrel{r_{n}}{\longrightarrow} \infty$ разность $K_{n}(x)-K_{n+N_{b}}(x)$ ограничена, а также $\left(\kappa_{n+N_{b}}-\kappa_{n}\right) x_{2} \rightarrow-\infty$, а при $x \stackrel{\sigma_{n}}{\longrightarrow} \infty$ мы имеем $K_{n+N_{b}-1}(x)-K_{n-1}(x) \rightarrow+\infty$ и $K_{n}(x)-K_{n+N_{b}}(x) \rightarrow+\infty$, что вытекает непосредственно из определения. На самом деле, можно доказать более общее утверждение.

Лемма 2. Пусть $N_{a}, N_{b} \geqslant 1 u n \in \mathbb{Z}$ произвольно. Тогда:

1) если $x \stackrel{r_{n}}{\longrightarrow} \infty$, то $K_{l}(x)-K_{m}(x)$ либо стремится $\kappa+\infty$, либо ограничено при любых $l=n, \ldots, n+N_{b}$ u $m=n+N_{b}, \ldots, \mathcal{N}+n$, причем ограниченность имеет место тогда и только тогда, когда пара индексов $(l, m)$ принимает значения $(n, n)$, $\left(n, n+N_{b}\right),\left(n+N_{b}, n\right),\left(n+N_{b}, n+N_{b}\right)$

2) если $x \stackrel{\sigma_{n}}{\longrightarrow} \infty$, mо $K_{l}(x)-K_{m}(x) \rightarrow+\infty$ для любых $l=n, \ldots, n+N_{b}-1 u$ $m=n+N_{b}, \ldots, \mathcal{N}+n-1$, где суммирование в индексах всегда понимается по модулю $\mathcal{N}$.

ДоказАтельство. Вследствие (3.1) и (3.4) имеем $K_{l}\left(y_{n}\right)-K_{m}\left(y_{n}\right)=g_{l, m, n}$. Тогда первое утверждение леммы следует непосредственно из определения и леммы 1. Аналогично получаем, что $K_{l}(x)-K_{m}(x)=\alpha g_{l, m, n-1}+\beta g_{l, m, n}$ при подстановке $x=\alpha y_{n-1}+\beta y_{n}$. Тогда второе утверждение леммы следует из второго утверждения леммы 1 , в то время как интервалы для $l$ и $m$ появляются как пересечения соответствующих интервалов в $(2.24)$ для $n$ и $n \rightarrow n-1$. Лемма доказана.

Теперь мы можем доказать следующую теорему.

Теорема. Асимптотическое поведение функиии $\tau(x)$ при $x \rightarrow \infty$ задается следующими соотношениями:

$$
\begin{aligned}
& x \stackrel{r_{n}}{\longrightarrow} \infty: \quad \tau(x)=\left(z_{n}+z_{n+1} e^{K_{N_{b}+n}(x)-K_{n}(x)}+o(1)\right) \exp \left(\sum_{j=n}^{n+N_{b}-1} K_{j}(x)\right), \\
& x \stackrel{\sigma_{n}}{\longrightarrow} \infty: \quad \tau(x)=\left(z_{n}+o(1)\right) \exp \left(\sum_{l=n}^{n+N_{b}-1} K_{l}(x)\right)
\end{aligned}
$$

для любого $n \in \mathbb{Z}$, где коэфбициенты $z_{n}$ определены как

$$
z_{n}=f_{n, \ldots, n+N_{b}-1} \equiv V\left(\kappa_{n}, \ldots, \kappa_{n+N_{b}-1}\right) \mathcal{D}\left(n, \ldots, n+N_{b}-1\right)
$$

(ср. (2.18)). При этом члены о(1) убъвают экспоненциально.

ДокАзАтЕльство. Используя представление (2.22), можно записать

$$
\begin{aligned}
\exp \left(-\sum_{l=n}^{n+N_{b}-1} K_{l}(x)\right) \tau(x)= \\
\quad=\sum_{n \leqslant n_{1}<\cdots<n_{N_{b}} \leqslant \mathcal{N}+n-1} f_{n_{1}, \ldots, n_{N_{b}}} \exp \left(\sum_{j=1}^{N_{b}} K_{n_{j}}(x)-\sum_{l=n}^{n+N_{b}-1} K_{l}(x)\right) .
\end{aligned}
$$


Рассмотрим сначала асимптотику при $x \stackrel{\sigma_{n}}{\longrightarrow} \infty$. Тогда каждый член $K_{n_{j}}(x)$ либо сокращается с некоторым членом в последней сумме, когда $n_{j}=l(\bmod \mathcal{N})$, где некоторый индекс $l$ принадлежит интервалу $\left\{n, \ldots, n+N_{b}-1\right\}$ (т. е. интервалу для индекса $l$ во втором утверждении леммы 2 ), либо индекс $n_{j}$ принадлежит интервалу $\left\{n+N_{b}, \ldots, \mathcal{N}+n-1\right\}$. Тогда по лемме 2 все эти показатели на бесконечности отрицательны за единственным исключением $\left\{n_{1}, \ldots, n_{N_{b}}\right\}=\left\{n, \ldots, n+N_{b}-1\right\}(\bmod \mathcal{N})$. Это доказывает (3.6). Доказательство асимптотики (3.5) проводится аналогично, с единственным отличием, что используется первое утверждение леммы 2 . Утверждение об асимптотическом поведении членов типа $о(1)$ очевидно по построению. Теорема доказана.

Как видно из соотношений (3.5) и (3.6), лидирующее асимптотическое поведение фиксируется этой теоремой, только если

$$
z_{n}>0, \quad n=1, \ldots, \mathcal{N} .
$$

Это условие достаточно для регулярности потенциала при больших $x$, но конкретные примеры показывают, что оно недостаточно для регулярности потенциала на всей плоскости $\left(x_{1}, x_{2}\right)$. Мы видим также, что асимптотика вдоль луча $r_{n}$ дается суммой лидирующих членов, полученных для $x \stackrel{\sigma_{n}}{\longrightarrow} \infty$ и $x \stackrel{\sigma_{n+1}}{\longrightarrow} \infty$. Поскольку экспоненциальный фактор исчезает при подстановке разложения (3.5) для $\tau(x)$ в $(2.4)$, множитель в скобках дает поведение потенциала $u(x)$ на бесконечности вдоль лучей. Итак, если наложено условие $(3.8)$, то потенциал $u(x)$ имеет в точности $\mathcal{N}$ лучей $r_{n}$, дающих нетривиальное асимптотическое поведение односолитонного потенциала (ср. с потенциалом (1.4)) с параметрами $\kappa_{n}$ и $\kappa_{n+N_{b}}$. Принимая во внимание (2.21), мы получаем в явном виде следующую структуру лучей и асимптотическое поведение вдоль лучей: если $x_{2} \rightarrow-\infty, x_{1}+\left(\kappa_{n}+\kappa_{n+N_{b}}\right) x_{2}$ ограничено, TO

$$
u(x) \cong-2 \partial_{x_{1}}^{2} \ln \left(z_{n}+z_{n+1} e^{K_{n+N_{b}}(x)-K_{n}(x)}\right), \quad n=1, \ldots, N_{a} ;
$$

если $x_{2} \rightarrow+\infty, x_{1}+\left(\kappa_{n}+\kappa_{n+N_{a}}\right) x_{2}$ ограничено, то

$$
u(x) \cong-2 \partial_{x_{1}}^{2} \ln \left(z_{n+N_{a}}+z_{n+N_{a}+1} e^{K_{n}(x)-K_{n+N_{a}}(x)}\right), \quad n=1, \ldots, N_{b},
$$

т. е. мы имеем $N_{a}$ асимптотических лучей в нижней полуплоскости и $N_{b}$ лучей в верхней полуплоскости. Асимптотическое поведение внутри секторов $\sigma_{n}$ дается соотношением (3.6), где единственный зависящий от $x$ член - это экспоненциальный множитель. Принимая во внимание его линейную зависимость от $x$ и условие (3.8), получаем, что $u(x)$ экспоненциально убывает по всем направлениям во всех сектоpax.

\section{4. ЗАКЛЮЧИТЕЛЬНЫЕ ЗАМЕЧАНИЯ}

Полученное в настоящей работе асимптотическое поведение тау-функции и потенциала основано на условии (3.8), допускающем обращение в ноль или отрицательные значения для коэффициентов в (2.17), отличных от $z_{n}$ (см. (3.7)). Используя условие (3.8), мы смогли найти, что направления лучей в явном виде даются суммами $\kappa_{n}+\kappa_{n+N_{b}}$ при $n=1, \ldots, \mathcal{N}$ и вывести точное асимптотическое поведение тау-функций на плоскости $\left(x_{1}, x_{2}\right)$. Отметим, что аналогичный результат был 
получен в работе [18] для очень специального подкласса солитонных потенциалов, задаваемых матрицей $\mathcal{D}$ в $(2.5)$, равной произведению диагональной матрицы на матрицу, транспонированную к $\mathcal{V}$. Как было отмечено выше, условие (3.8), в противоположность (2.20), не гарантирует отсутствие сингулярностей потенциала $u(x)$. Но формулы (3.5) и (3.6) доказывают, что в силу (3.8) сингулярности потенциала (2.4) не могут появиться в асимптотике, поскольку внутри секторов потенциал $u(x)$ стремится к нулю, а вдоль лучей $r_{n}$ он ведет себя в соответствии с (3.9) и (3.10) и также конечен в силу (3.8). Подчеркнем, что параметры $N_{a}$ и $N_{b}$, которые задают числа лучей тау-функции и потенциала в соответственно нижней и верхней полуплоскостях, появились в работе [10] как числа полюсов решения Йоста и дуального решения Йоста. И, наконец, отметим, что доказанная в настоящей работе теорема также верна, когда все неравенства в условии регулярности $(2.20)$ являются строгими, т. е. все максимальные миноры матрицы $\mathcal{D}$ положительны, поскольку это условие сильнее, чем (3.8), которое было достаточно для доказательства теоремы.

Благодарности. Работа частично поддержана РФФИ (гранты № 08-01-00501, 09-01-92433-КЭ), Программой поддержки ведущих научных школ (грант НШ-8265. 2010.1), Программой РАН "Фундаментальные проблемы нелинейной динамики", INFN (грант PRIN 2008 "Geometrical methods in the theory of nonlinear integrable systems") и Консорциумом E.I.N.S.T.E.I.N. A. K. Погребков благодарит факультет физики Университета Саленто (Лечче) за гостеприимство.

\section{Список литературы}

[1] Б. Б. Кадомцев, В. И. Петвиашвили, Докл. АН СССР, 192 (1970), 753-756.

[2] В. Е. Захаров, А. Б. Шабат, Функи. анализ и его прил., 8:3 (1974), 43-53.

[3] В. С. Дрюма, Писъма в ЖЭТФ, 19:12 (1974), 753-755.

[4] M. J. Ablowitz, D. Bar Yaacov, A. S. Fokas, Stud. Appl. Math., 69:2 (1983), 135-143.

[5] В. Д. Липовский, Функи. анализ и его прил., 20:4 (1986), 35-45.

[6] M. V. Wickerhauser, Comm. Math. Phys., 108:1 (1987), 67-89.

[7] П. Г. Гриневич, С. П. Новиков, Функи. анализ и его прил., 22:1 (1988), 23-33.

[8] M. Boiti, F. Pempinelli, A. K. Pogrebkov, J. Math. Phys., 47:12 (2006), 123510, 43 pp.

[9] M. Boiti, F. Pempinelli, A. K. Pogrebkov, B. Prinari, J. Math. Phys., 43:2 (2002), 1044-1062.

[10] М. Бойти, Ф. Пемпинелли, А. К. Погребков, Б. Принари, ТМФ, 159:3 (2009), 364-378, arXiv: 0901.3857.

[11] M. Boiti, F. Pempinelli, A. Pogrebkov, B. Prinari, Inverse Problems, 17:4 (2001), 937-957.

[12] М. Бойти, Ф. Пемпинелли, А. К. Погребков, Б. Принари, ТМФ, 165:1 (2010), 3-24, arXiv: 0911.1675.

[13] Y. Kodama, J. Phys. A, 43:43 (2010), 434004, 54 pp., arXiv: 1004.4607.

[14] G. Biondini, S. Chakravarty, Math. Comput. Simulation, 74:2-3 (2007), 237-250.

[15] Ф.Р. Гантмахер, Теория матрии, Наука, М., 1988.

[16] T. Ando, Linear Algebra Appl., 90 (1987), 165-219.

[17] Ф.Р. Гантмахер, М.Г. Крейн, Осцилляционные матрицы и ядра, и малые колебания механических систем, ГТТИ, М., Л., 1950.

[18] G. Biondini, Y. Kodama, J. Phys. A, 36:42 (2003), 10519-10536, arXiv: nlin/0306003. 